\title{
Neutralizing Ceramide: A Major Breakthrough or Yet Another Marginal Target for the Treatment of Acute Lung Injury?
}

\begin{abstract}
A review of Göggel R, Winoto-Morbach S, Vielbaher G, Imai Y, Lindner K, Brade L, Brade H, Ehlers S, Slutsky AS, Schütze S, Gulbins E, Uhlig S 2004 PAF-induced pulmonary edema: a new role for acid sphingomyelinase and ceramide. Nat Med 10:155-160.
\end{abstract}

$\mathrm{A}$ cute lung injury (ALI) is a complication of various disorders, including toxic inhalation, viral pneumonia, and sepsis, that literally drowns patients suffering from these conditions. The physiopathological mechanisms that cause alveolar flooding, which effectively impair gas exchange, were unraveled many years ago (1). However, the intricate cellular and molecular mechanisms underlying alveolar flooding remain elusive, and the search for an understanding of how and when lung cells begin losing their normal function, becoming weak and leaky, remains a vital quest.

Many mediators have been implicated in ALI and the acute respiratory distress syndrome (ARDS), including tumor necrosis factor- $\alpha$ (TNF- $\alpha$ ), platelet-activating factor (PAF) and prostaglandins (2). Because there are probably several interacting mechanisms involved, blocking a single mediator has not proven effective against ALI in clinical trials. In many experimental models of ALI, edema formation is mediated by PAF (3). Moreover, application of recombinant PAF acetylhydrolase markedly decreases vascular leakage in animal models (4), and has shown promising results in phase 2 clinical trials for severe sepsis (5).

The molecular mechanisms by which PAF triggers edema formation are poorly understood. Using the isolated, perfused lung, edema formation can be easily monitored by measuring lung weight. In addition, the two confounding factors observed in the in vivo model of pulmonary hypertension

\section{Jean-Christophe Mercier,}

\section{A. Tuan Dinh-Xuan}

are excluded. First, by perfusing the lungs with constant hydrostatic pressure, the confounding effects of pulmonary hypertension are excluded, and second by using of a blood-free perfusate, blood-borne leukocytes are eliminated. Using this model, PAF was recently shown to trigger edema by increasing microvascular permeability through two independent mechanisms. One pathway involves activation of EP-3 receptors by prostaglandin $E_{2}$, which can be blocked by cyclooxygenase inhibitors such as acetyl salicylic acid (ASA) (6). The nature of the other pathway is elusive. Based on the observation that PAF stimulates ceramide formation in mouse macrophages (7), the authors hypothesized that ceramide is involved in PAFinduced edema formation.

Ceramide ( $\mathrm{N}$-acyl-sphingosine) is a lipid that lies within the cell membrane bilayer, forming the backbone for glycosphingolipids. More than a simple hydrophobic moiety anchoring glycolipid molecules in the membrane bilayer, ceramide is also known to function as a signaling molecule (Fig. 1). Ceramide is produced from membrane sphingomyelin by several sphingomyelinases, one of which is the secretory acid sphingomyelinase (SA-SMase), which is activated by several inflammatory mediators including PAF. There are at least two alternative pathways that can be followed by ceramide generated at the outer leaflet of the membrane. Either ceramide flips inwards and undergoes hydrolysis by ceramidase to generate sphingosine, which is, in turn, reversibly phosphorylated by sphingosine kinase to give sphingosine-1-phosphate (S1P). Alternatively, sphingomyelin synthase catalyzes the transfer of the phosphorylcholine moiety from one molecule of phosphatidylcholine to one molecule of ceramide to form one molecule of sphingomyelin and one molecule of diacylglycerol (DAG) (8). DAG activates protein kinase $\mathrm{C}$ (PKC). Both $\mathrm{PKC}$ and sphingosine-1-phosphate can activate the transcription factor, nuclear factor $-\kappa \mathrm{B}(\mathrm{NF}-\kappa \mathrm{B})$. Note that the enzyme sphingosine kinase can be activated by $\mathrm{TNF} \alpha$, thus providing another mean to stimulate S1P by inflammatory mediators $(9,10)$. Mediators derived from ceramide can also cause apoptosis (11), either by means of S1P formation or through activation of DAG-PKC pathway, hence further worsening the tissue damage that was already triggered by inflammatory processes.

In the present study, SA-SMasedeficient or wild-type mice were treated with PAF. Following PAF administration, normal mice developed pulmonary edema within $10 \mathrm{~min}$; whereas, in SA-SMase-deficient mice, or wild-type mice pretreated with ASA, pulmonary edema was reduced by $\sim 50 \%$. Treatment of SA-SMasedeficient mice with ASA completely eliminated PAF-induced edema, indicating that PAF-induced edema is mediated by the combined action of SA- 


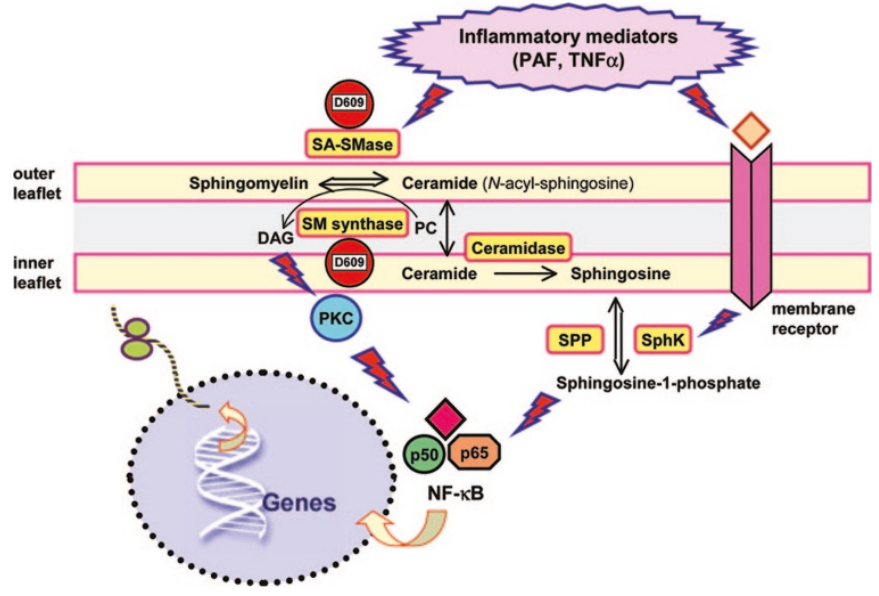

Figure 1. The sphingomyelinase - ceramide pathway in the inflammatory response of the lung. Ceramide is produced from membrane sphingomyelin (SM) by the secretory acid sphingomyelinase (SA-SMase) enzyme, which is activated by several inflammatory mediators including plateletactivating factor (PAF). There are at least two alternative pathways that can be followed by ceramide generated at the outer leaflet of the membrane. Either ceramide flips inwards and undergoes hydrolysis by ceramidase to generate sphingomyelin, which is, in turn, reversibly phosphorylated by sphingosine kinase (SphK) to give sphingosine-1-phosphate (S1P). Alternatively, SM synthase catalyzes the transfer of the phosphorylcholine moiety from one molecule of phosphatidylcholine (PC) to one molecule of ceramide to form one molecule of sphingomyelin and one molecule of diacylglycerol (DAG). DAG activates protein kinase C (PKC). Both PKC and S1P can activate NF- $\kappa$ B. Note that D069 can inhibit both SA-SMASE and SM synthase. Also note that the enzyme sphingosine kinase (SphK) can be activated by tumor necrosis factor- $\alpha$ (TNF- $\alpha$ ), thus providing another mean to stimulate S1P by inflammatory mediators. DAG, diacylglycerol; NF-kappa B, nuclear factor kappa B; PC, phosphatidylcholine; PKC, protein kinase C; SA-Smase, secretory acid sphingomyelinase; SM synthase, sphingomyelin synthase, SPP, sphingosine-1-phosphate phosphatase, SphK, sphingosine kinase.

SMase and cyclo-oxygenase. Furthermore, perfusion of rat lungs with PAF rapidly increased the activity and amount of SA-SMase, demonstrating that PAF triggers ASM release from the lungs. Because SA-SMase hydrolyses SM to ceramide, the concentrations of ceramide in the lungs of PAF-treated mice were determined. PAF treatment increased the tissue concentration of long-chain ceramides by $\sim 50 \%$, whereas no such an increase was observed in SA-SMase-deficient mice. As long-chain ceramides are insoluble in the perfusate, perfusion of rat lung with $\mathrm{C} 2$-ceramide $(50 \mu \mathrm{M})$ caused severe edema. Taken together, these findings indicate a central role of SA-SMase-derived ceramide in PAFinduced pulmonary edema. However, as it is unlikely to achieve in human patients the very high concentration of $\mathrm{C} 2$-ceramide that was used by the authors to induce experimental edema in mice, interpretation of experimental results from this study and their application to clinical situation should be made with caution.
To neutralize surface ceramide in the outer leaflet of the cell membrane, two ceramide-specific antisera were studied on PAF-induced edema formation. Antisera prevented PAF- triggered pulmonary edema but had no effect on other PAF actions, such as the decreases in pulmonary vasoconstriction and bronchoconstriction. The effects of two different pharmacologic inhibitors, xanthogenate D609 and imipramine, were tested. Pretreatment with either of these agents prevented the PAF-induced increase in pulmonary ceramide and attenuated the development of PAF-induced edema. To extend these in vitro findings to whole intact animals, mice were pretreated with D609 and then challenged with PAF. Pretreatment of mice with D609 prevented PAF-induced edema in vivo. Because steroids may lower ceramide synthesis, the effect of dexamethasone (DXM) on PAF-induced ceramide and edema formation was investigated in mice in vivo. Pretreatment of mice with DXM prevented the increase in pulmonary ceramide and attenuated edema formation. Finally, D609pretreated mice or rats were partly resistant to the pulmonary edematous effects of endotoxin and acid instillation.

How well might the new data apply to humans? Plasma ceramide is increased in septic patients and correlates with mortality (12). Ceramide derivatives are markedly elevated in bronchoalveolar lavage fluid of patients with ARDS (13). A number of therapies tested for ARDS may interfere with the ceramide pathway, but not effectively. Steroids provide little or no clinical benefit in ARDS, although they partially reduce the generation of ceramide. However promising might be the study performed by Göggel and colleagues, one important issue that has been left out by the authors should be remembered here. While the proteolytic effects of imipramine on SA-SMase are undisputed, mechanisms underlying the effects of D609 on ceramide pathways are still a matter of controversies. The assumption made by Göggel and colleagues that D069 mainly acts as an inhibitor of SASMase should be weighted up against opposite findings suggesting that D609 might also act as an inhibitor of SMsynthase (8). Depending on the underlying mechanisms that are involved, the spotlight can either be directed toward the DAG-PKC pathways or to signaling molecules acting downstream ceramide formation. Although promising, the role of ceramide in ALI/ARDS can only be established when specific inhibitors of sphingomyelin pathways are available for further investigations.

\section{REFERENCES}

1. Rinaldo JE, Rogers RM 1982 Adult respiratorydistress syndrome: changing concepts of lung injury and repair. N Engl J Med 306:900-909

2. Ware LB, Matthay MA 2000 The acute respiratory distress syndrome. N Engl J Med 342:1334-1349

3. Nagase T, Ishii S, Kume K, Uozumi N, Izumi T, Ouchi Y, Shimizu T 1999 Platelet-activating factor mediates acid-lung injury in gentically engineered mice. J Clin Invest 104:1071-1076

4. Tjoeker LW, Wilder C, Eberhardt C, Stafforinit DM, Dietsch G, Schimpf B, Hooper S, Le Trong H, Cousens LS, Zimmerman GA, Yamadat Y, Mclntyre TM, Prescott SM, Gray PW 1995 Antiinflammatory properties of platelet-activating factor acetyl-hydrolase. Nature 374:549-553

5. Schuster DP, Metzler M, Opal S, Lowry S, Balk R, Abraham E, Levy H, Slotman G, Coyne E, Souza S, Pribble J 2003 Recombinant platelet-activating factor acetylhydrolase to prevent acute respiratory distress 


\section{RESEARCH NEWS}

syndrome and mortality in severe sepsis : phase IIb, multicenter, randomised, placebo-controlled, clinical trial. Crit Care Med 31:1612-1619

6. Falk S, Goggel R, Heydasch U, Brasch F, Muller KM, Wendel A, Uhlig S 1999 Quinolines attenuate PAF-induced pulmonary pressor responses and edema formation. Am J Resp Crit Care Med 160:1734-1742

7. Balsinde J, Balboa MA, Dennis EA 1997 Inflammatory activation of arachidonic acid signaling in murine P388D1 macrophages via sphingomylin synthesis. J Biol Chem 272:20273-20377

8. Luberto C, Hannun YA 1998 Sphingomyelin synthase, a potential regulator of intracellular levels of ceramide and diacylglycerol during SV40 transformation. Does sphingomyelin synthase account for the putative phosphatidylcholine-specific phospholipase C? J Biol Chem 273:14550-14559

9. Spiegel S, Milstien 2003 Sphingosine-1-phosphate: an enigmatic signalling lipid. Nat Rev Mol Cell Biol 4:397-407

10. Saba JD, Hla T 2004 Point-counterpoint of sphingosine-1-phosphate metabolism. Circ Res 94:724734

11. Dbaibo GS, Hannun YA 1998 Signal transduction and the regulation of apoptosis: roles of ceramide. Apoptosis 3:317-334

12. Drobnik W, Liebisch G, Audebert FX, Frohlich D, Gluck T, Vogel P, Rothe G, Schmitz G 2003 Plasma ceramide and lysophosphatidylcholine inversely correlate with mortality in sepsis patients. J Lipid Res 44:754-761
13. Rauvala H, Hallman M 1984 Glycolipid accumulation in bronchoalveolar space in adult respiratory distress syndrome J Lipid Res 25:1257-1262

Service de Réanimation Pédiatrique Hôpital Robert Debré

Paris, France

jean-christophe.mercier@rdb.ap-hop-paris.fr Service de Physiologie

Hôpital Cochin

Paris, France

DOI: 10.1203/01.PDR.0000153948.44951.5B 